\title{
Review: pressurised metered dose inhalers are as effective as other hand held inhalers for corticosteroid use in asthma
}

\author{
Brocklebank D, Wright J, Cates C, on behalf of the National Health Technology Assessment Inhaler Review Group. Systematic \\ review of clinical effectiveness of pressurised metered dose inhalers versus other hand held inhaler devices for delivering \\ corticosteroids in asthma. BMJ 2001 Oct 20;323:896-900.
QUESTION: In patients with stable asthma, is the standard chlorofluorocarbon containing pressurised metered dose inhaler (PMDI) as effective as other hand held inhaler devices for delivering corticosteroids?

\section{Data sources}

Studies published from 1966 to July 1999 were identified by searching the Cochrane Airways Group trials database (derived from Medline, EMBASE/ Excerpta Medica, CINAHL; hand searches of 18 relevant journals and proceedings of 3 respiratory societies; and reviews of bibliographies of relevant studies). Pharmaceutical companies that manufacture inhaled asthma drugs were contacted for unpublished studies.

Source of funding:
NHS Research and
Development Health
Technology Assessment
Programme.
For correspondence:
DrJ Wright, Bradford
Hospitals NHS Trust,
Bradford,UK.
john.wright@
bradfordhospitals.nhs.uk.
A modified version of
this abstract also
appears in
Evidence-Based
Nursing.

\section{Study selection}

Studies in any language were selected if they were laboratory, hospital, or community based randomised controlled trials of children or adults that lasted $\geqslant 4$ weeks and compared a single drug delivered by a standard PMDI (with or without a spacer) with any other hand held inhaler. Trials comparing different doses of the same drug were also included.

\section{Data extraction}

2 reviewers independently extracted data on study design, patient characteristics, details of the intervention, study duration, outcomes, and quality. Outcomes included lung function $\left(\mathrm{FEV}_{1}\right)$, quality of life measurements, symptom

Pressurised metered dose inhaler $v$ dry powder inhaler for delivering corticosteroids in asthma (parallel studies only)*

\begin{tabular}{lll} 
Outcomes at $\geqslant 4$ weeks & Number of studies $(\mathrm{n})$ & SMD $(95 \% \mathrm{Cl})$ \\
\hline FEV & $7(1404)$ & $-0.14(-0.25$ to -0.03$)$ \\
\hline Morning peak expiratory flow rate & $7(1389)$ & $-0.14(-0.25$ to -0.04$)$ \\
\hline Use of additional relief drugs & $6(967)$ & $-0.18(-0.31$ to -0.05$)$ \\
\hline
\end{tabular}

*SMD = standardised mean difference; minus sign means results favour the dry powder inhaler. A fixed effects model was used. scores, drugs for additional relief, acute exacerbation, days off work or school, treatment failure, patient compliance, patient preference, adverse effects, bronchial hyperreactivity, and systemic bioavailability.

\section{Main results}

24 articles describing 29 studies met the selection criteria. 14 studies compared PMDIs with dry powder inhalers (DPIs): PMDIs were less effective than DPIs for improving $\mathrm{FEV}_{1}$, morning peak expiratory flow rate, and use of additional relief drugs (table). However, these differences in improvement either disappeared after adjusting for baseline variables or were within clinically equivalent limits. 11 studies compared chlorofluorocarbon PMDI with hydrofluoroalkane (10 studies used beclomethasone, and 1 study used fluticasone). Treatment effects did not differ. 1 study compared breath actuated PMDI with PMDI but found no differences for any outcomes. 3 studies of children were included, but a meta-analysis of the results could not be done because of study differences. None of the studies of children found any differences in pulmonary function between the devices. However, 1 study found that the Turbohaler group reduced their use of relief drugs by 1 puff per week (95\% CI 0.35 to 1.96$)$ more than the PMDI group.

\section{Conclusion}

In patients with stable asthma, the standard chlorofluorocarbon containing pressurised metered dose inhaler is as effective as other hand held inhaler devices for delivering corticosteroids.

\section{COMMENTARY}

PMDIs have been used since the early 1990s to deliver aerosolised $\beta_{2}$ agonists and corticosteroids to the lung. In recent years, environmental concern over chlorofluorocarbon propellants has fuelled interest in the development of alternative delivery systems. The reviews by Brocklebank $e t$ al and Ram $e t$ al found no difference between PMDIs and other hand held delivery systems in patients with stable mild to moderate asthma. They concluded that the standard PMDI remains the most cost effective delivery device.

Both device specific and patient specific issues influence the success or failure of a particular delivery system. Patient factors influencing drug delivery and therapeutic success include not only coordination but also breath holding ability and inspiratory flow rate, both influenced by disease severity. Findings in patients with mild to moderate asthma may not be applicable to patients with more severe airway obstruction.

Proper education in the use of the various hand held delivery systems is crucial because substantial rates (between 12\% and 90\%) of incorrect metered dose inhaler use have been reported. ${ }^{1}$ In the investigational setting (the "best case scenario") patients receive detailed instructions that are reinforced throughout the study. "Real world" patients receive varying degrees of instruction, and adherence to correct technique, if not reinforced, often wanes in the long term. Psychosocial factors (cultural beliefs, education, and language skills) may also influence the ability to comprehend and follow instructions.

The available delivery systems each have advantages and disadvantages. PMDIs are portable and convenient, but effectiveness is technique dependent. Furthermore, excessive oropharyngeal deposition may lead to local side effects with inhaled corticosteroids. Spacer devices can improve PMDI efficiency and decrease toxicity by decreasing particle size and increasing the lung : throat deposition ratio, but they are cumbersome to use and vary in the efficiency of drug delivery. Their main advantage is in reducing problems with lack of coordination between actuation and inhalation. 


\section{Review: pressurised metered dose inhalers are as effective as other hand held inhalers for $\beta_{2}$ agonist bronchodilator use in asthma}

Ram FS, Wright J, Brocklebank D, et al, on behalf of the National Health Technology Assessment Inhaler Review Group. Systematic review of clinical effectiveness of pressurised metered dose inhalers versus other hand held inhaler devices for delivering $\beta_{2}$ agonist bronchodilators in asthma. BMJ 2001 Oct 20;323:901-5.

QUESTION: In patients with stable asthma, is the standard chlorofluorocarbon containing pressurised metered dose inhaler (PMDI) as effective as other handheld inhaler devices, including chlorofluorocarbon free PMDIs, for delivering $\beta_{2}$ agonist bronchodilators?

\section{Data sources}

Studies published from 1966 to December 2000 were identified by searching the Cochrane Airways Group trials database (derived from Medline, EMBASE/ Excerpta Medica, CINAHL; handsearches of 20 relevant journals and proceedings of 3 respiratory societies; and review of the bibliographies of included trials). Medline, EMBASE/Excerpta Medica, CINAHL, and 17 online respiratory websites were also independently searched, and pharmaceutical companies that manufacture inhaled asthma drugs were contacted for unpublished studies.

\section{Study selection}

Studies in any language were selected if they were laboratory, hospital, or community based randomised controlled trials of children or adults that compared delivery of $\beta_{2}$ agonist bronchodilators by standard PMDI (with or without a spacer) with any other hand held inhaler. Trials comparing different doses of inhaled drug and those that used challenge testing were also included.

\section{Data extraction}

2 reviewers independently extracted data on study design, patient characteristics, details of the intervention, study duration, outcomes, and quality. Outcomes included lung function $\left(\mathrm{FEV}_{1}\right)$, quality of life measurements, symptom scores, drugs for additional relief, steroid requirements, nocturnal awakening, acute exacerbations, days off work or school, treatment failures, patient compliance, patient preferences, adverse effects, bronchial hyperreactivity, and systemic bioavailability.

\section{Main results}

89 articles describing 84 studies met the selection criteria. 71 trials involved adults and 13 involved children. In most trials, patients had mild to moderate asthma (baseline $\mathrm{FEV}_{1}>50 \%$ of predicted). Meta-analyses were done using a fixed effects model. In both adults and children, standard PMDIs did not differ from any of the other 10 hand held inhaler devices (Turbohaler, Diskhaler, hydrofluoroalkane PMDI, Rotahaler, Spiros, Easyhaler, multidose powder inhaler, Clickhaler, Gentlehaler, and Autohaler) for $\mathrm{FEV}_{1}$, forced vital capacity, peak expiratory flow rate, area under the curve for $\mathrm{FEV}_{1}$, blood pressure, symptoms, bronchial hyperreactivity, systemic bioavailability, inhaled steroid requirement, serum potassium concentration, or use of additional relief brochodilators. However, regular use of the hydrofluoralkane PMDI containing salbutamol was associated with reduced requirement for short courses of oral corticosteroids (relative risk $0.67,95 \%$ CI 0.49 to 0.91 ).

\section{Conclusion}

In patients with stable asthma, the standard chlorofluorocarbon containing pressurised metered dose inhaler (PMDI) is as effective as other hand held inhalers, including chlorofluorocarbon free PMDIs, for delivering $\beta_{2}$ agonist bronchodilators.

\section{Source of funding: NHS Research and Development Health Technology Assessment Programme.}

For correspondence: Dr J Wright, Bradford Hospitals NHS Trust, Bradford, UK john.wright@ bradfordhospitals.nhs.uk

A modified version of this abstract also appears in Evidence-Based Nursing.

\section{COMMENTARY_continued from previous page}

The amount of aerosol delivered to the lungs is determined by aerosol particle size, velocity, and inspiratory flow rate. In the hydrofluoroalkane PMDIs, the drug is reformulated as a solution. This approach increases the fine particle fraction, which results in increased drug delivery to the lung and decreased oropharyngeal deposition. Hydrofluoroalkane PMDIs are portable, convenient, and contain no chlorofluorocarbon propellants.

DPI systems are a portable, propellant free, breath actuated alternative to PMDIs that help overcome breath to hand coordination difficulties. Many have dose counters that allow patients and physicians to monitor treatment. However, in outpatient settings, delivery that requires loading may be inconvenient and may result in decreased adherence to treatment. The efficiency of drug delivery with DPIs also varies according to a patient's inspiratory flow rate.

With all hand held delivery systems, drug delivery and efficacy are affected by such patient factors as coordination, inspiratory flow rate, and technique. As clinicians, our primary goal is to enhance compliance with treatment for optimal drug delivery while keeping overall costs low. To maximise treatment success, the choice of a delivery system must take into account patient preference, ability, lifestyle, and disease severity. Cost effectiveness will be maximised with a delivery device that is tailored to a patient's needs and abilities because reduced adherence may result from lack of perceived efficacy.

These 2 reviews provide evidence that, in an ideal setting, PMDIs can be as effective as newer technologies developed to overcome pitfalls in patient technique and compliance.

Amy R Blanchard, MD Cleveland Clinic Foundation, Cleveland, Ohio, USA Joseph A Golish, MD Medical College of Georgia, Augusta, Georgia, USA

1 McFadden ER Jr. Improper patient techniques with metered dose inhalers: clinical consequences and solutions to misuse. J Allergy Clin Immunol 1995;96:278-83. 